(c) 2010 IEEE. Personal use of this material is permitted. Permission from IEEE must be obtained for all other uses, in any current or future media, including reprinting/republishing this material for advertising or promotional purposes, creating new collective works, for resale or redistribution to servers or lists, or reuse of any copyrighted component of this work in other works. 


\section{Allpass VFD Filter Design}

Hai Huyen Dam and Kok Lay Teo

\begin{abstract}
This correspondence proposes a general design for allpass variable fractional delay (VFD) digital filters with minimum weighted integral squared error subject to constraints on maximum error deviation from the desired response. The resulting optimization problem is nonlinear and nonconvex with a nonlinear continuous inequality constraint. Stability of the designed filters are discussed. An effective procedure is proposed for solving the optimization problem. Firstly, a constraint transcription method and a smoothing technique are employed to transform the continuous inequality constraint into one equality constraint. Then, by using the concept of a penalty function, the transformed constraint is incorporated into the cost function to form a new cost function. The nonlinear optimization problem subject to continuous inequality constraints is then approximated by a sequence of unconstraint optimization problems. Finally, a global optimization method using a filled function is employed to solve the unconstraint optimization problem. Design example shows that a trade-off can be achieved between the integral squared error and the maximum error deviation for the design of allpass VFD filters.
\end{abstract}

Index Terms - Allpass filter design, filled function, optimization, variable digital filter design.

\section{INTRODUCTION}

Digital filters with tunable fractional phase-delay or fractional group delay, referred to as variable fractional-delay (VFD) filters, are useful in various signal processing applications [1]-[3], including timing offset recovery in digital receivers, comb filter design, sampling rate conversion, speech coding and synthesis, time delay estimation, one-dimensional digital signal interpolation and image interpolation. For finite impulse response (FIR) based VFD filters, it is relatively easy to formulate and solve as an appropriate optimization problem so as to achieve the desired characteristics [1], [4], [5]. The design of allpass VFD filters is somewhat more difficult and has been investigated in [3], [6]-[9]. The advantage of the allpass VDF filters is that they can achieve higher design accuracy than FIR filters in terms of frequency response errors for applications that require unity gain.

In [3], [7], the design of an allpass VFD filter with minimum integral squared error has been investigated. However, the designs obtained often have high maximum error variation from the desired response. In this correspondence, we formulate a design for allpass VFD filters with minimum weighted integral square error subject to constraint on maximum error deviations from the desired response. Stability of the designed filters are also discussed. The formulated optimization problem is nonlinear and nonconvex with a nonlinear continuous inequality constraint. The optimization procedure for solving this problem is as follows. Initially, a constraint transcription method is employed to reduce the continuous inequality constraint into an equality constraint. As the equality constraint is non smooth, a local smoothing technique is used to smoothen it. Then, the transformed constraint is appended to the cost

Manuscript received July 23, 2009; accepted March 02, 2010. Date of publication April 15, 2010; date of current version July 14, 2010. The associate editor coordinating the review of this manuscript and approving it for publication was Dr. Soontorn Oraintara.

The authors are with Department of Mathematics and Statistics, Curtin University of Technology, Perth, 6102, Australia (e-mail: H.Dam@curtin.edu.au; K.L.Teo@curtin.edu.au).

Color versions of one or more of the figures in this paper are available online at http://ieeexplore.ieee.org.

Digital Object Identifier 10.1109/TSP.2010.2048316 function, forming a new cost function. In this way, the optimization problem with continuous inequality constraint is approximated by a sequence of unconstrained optimization problems [10]. A local search method is first used to solve these unconstrained optimization problems. Then, a global optimization method employing a filled function is used to escape from the local minimum obtained by the local search method to a lower basin in the search space [11]. This process is repeated until no lower basin can be found.

The design example that will be presented shows that a trade-off can be achieved between the integral squared error and the maximum error deviation. In addition, from the weighted least square solution, the maximum error deviation can be reduced while still maintaining approximately the same integral squared error.

The rest of the correspondence is organized as follows. The problem formulation is given in Section II. The approximation of the optimization problem with continuous inequality constraint by a sequence of unconstrained optimization problems is developed in Section III. The unconstrained optimization problem is solved by using an optimization procedure combining a local search method and a filled function in Section IV. Design examples are reported in Section V and concluding remarks are given in Section VI.

\section{PROBLEM Formulation}

In this section, we formulate a general design of an allpass VFD filter. The transfer function of the allpass filter is given by

$$
H(j \omega, p)=e^{-j N \omega} \frac{1+\sum_{n=1}^{N} a_{n}(p) e^{j n \omega}}{1+\sum_{n=1}^{N} a_{n}(p) e^{-j n \omega}}=e^{-j N \omega} \frac{A(-j \omega, p)}{A(j \omega, p)}
$$

where $a_{n}(p), 1 \leq n \leq N$, are allpass filter coefficients depending on the tuning parameter $p$, and

$$
A(j \omega, p)=1+\sum_{n=1}^{N} a_{n}(p) e^{-j n \omega} .
$$

The frequency $\omega \in \Omega=[0, \alpha \pi]$ with $0 \leq \alpha \leq 1$ and the tuning parameter $p$ is varied in the range

$$
\mathcal{P}=[-0.5,0.5]
$$

Each coefficient $a_{n}(p)$ is expressed in terms of an $M$ th order polynomial in terms of the parameter $p$ [3], i.e.,

$$
a_{n}(p)=\sum_{m=0}^{M} h_{n, m} p^{m}
$$

where $h_{n, m}$ are constant real coefficients. Similar to [3], we consider the case with $h_{n, 0}=0$ for all $1 \leq n \leq N$.

Let $\mathbf{h}=\left[h_{1,1}, \ldots, h_{1, M}, \ldots, h_{N, 1}, \ldots, h_{N, M}\right]^{T}$ be the $M N \times$ 1 vector of the allpass VFD filter coefficients. The desired frequency response of the allpass VFD filter is given by

$$
H_{d}(j \omega, p)=e^{-j \theta_{d}(\omega, p)}=e^{-j \tau_{d}(p) \omega}
$$

where $\theta_{d}(\omega, p)$ and $\tau_{d}(p)$ denote, respectively, the desired phase and the positive desired group delay, depending on the tuning parameter $p$. Here, $\tau_{d}(p)=I+p$ where $I$ is a constant. We design the allpass VFD filter with minimum integral squared error, defined as the integration of the weighted squared error deviation between $H(j \omega, p)$ and $H_{d}(j \omega, p)$, i.e.,

$$
E(\mathbf{h})=\int_{\mathcal{P}} \int_{\Omega} W(\omega, p)\left|H(j \omega, p)-H_{d}(j \omega, p)\right|^{2} d \omega d p
$$


where $W(\omega, p)$ is a positive weighting function, depending on the frequency $\omega$ and $p$. The filter is designed subject to a constraint on the peak error deviation between $H(\omega, p)$ and $H_{d}(\omega, p)$, i.e.,

$$
\left|H(\omega, p)-H_{d}(\omega, p)\right| \leq e(\omega, p), \forall \omega \in \Omega, p \in \mathcal{P}
$$

where $e(\omega, p)>0$ represents the allowed tolerance. Thus, the design of the allpass filter can be formulated as the following optimization problem:

$$
\begin{aligned}
& \min _{\mathbf{h} \in \mathcal{R} M N \times 1} E(\mathbf{h}) \\
& \text { subject to } \\
& \left|H(\omega, p)-H_{d}(\omega, p)\right| \leq e(\omega, p), \forall \omega \in \Omega, p \in \mathcal{P} .
\end{aligned}
$$

Here, we concentrate mainly on the investigation of the trade-off between the integral squared error and the maximum error deviation from the desired response.

Denote by $\theta(\omega, p)$ the phase response of the allpass filter $H(z, p)$. For a stable allpass filter, the phase $\theta(\omega, p)$ has the following three properties [8]: i) $\theta(0, p)=0$ for $p \in[-0.5,0.5]$; ii) $\theta(\pi, p)=-N \pi$ for $p \in[-0.5,0.5]$; and iii) $\theta(\omega, p)$ decreases monotonically with respect to the frequency $\omega$ for $p \in[-0.5,0.5]$. Since $\theta_{d}(0, p)=0$, $\theta_{d}(\pi, p)=-\pi(I+p)$ and $\theta_{d}(\omega, p)$ is a decreasing function, we choose $N=I$ so that properties i) and ii) are satisfied. Although the phase of the allpass filter follows closely a monotonically decreasing function, it does not imply that the phase itself is monotonic. The designed allpass filter is stable if it has a phase approximation error less than $\pi$ at $\omega=\pi$ for $p \in[-0.5,0.5]$ [8]. It has been noted in [7] that if $\alpha>0.85$ then $\theta(\pi, p)=-N \pi$ for all $p$. Thus, the phase error at $\omega=\pi$ is $\theta(\pi, p)-\theta_{d}(\pi, p)=p \pi$ which is less than $\pi$ at $\omega=\pi$. Hence, the designed allpass filters are stable and hence stability constraints are not required to be included in this case. For other cases, e.g., other ranges of $p$, additional stability constraints such as the sufficient stability condition given in [12] should be included in the optimization problem. Similar approach as the one proposed here can be used for solving the resulting optimization problem with two continuous inequality constraints.

\section{APPROXIMATE UNCONSTRAINED OPTIMIZATION PROBLEMS}

The optimization problem (6) is nonlinear and nonconvex, subject to continuous inequality constraints. Let the continuous inequality constraint in (6) be expressed as

$$
g(\mathbf{h}, \omega, p) \leq 0, \forall \omega \in \Omega, p \in \mathcal{P}
$$

where

$$
g(\mathbf{h}, \omega, p)=\left|H(j \omega, p)-H_{d}(j \omega, p)\right|^{2}-e^{2}(\omega, p) .
$$

The continuous inequality constraint in (6) is equivalent to the following equality constraint:

$$
G(\mathbf{h})=0
$$

where

$$
G(\mathbf{h})=\int_{\mathcal{P}} \int_{\Omega} \max \{g(\mathbf{h}, \omega, p), 0\} d \omega d p .
$$

The function $\max \{g(\mathbf{h}, \omega, p), 0\}$ in (9) is highly nonlinear. It is continuous with respect to $\mathbf{h}$ for every $\omega$ and $p$. Moreover, it is not differentiable and hence nonsmooth in terms of $\mathbf{h}$. Thus, standard gradient based optimization routines would have difficulties with this type of equality constraint. To overcome this problem, a smoothing technique [10] is employed to replace $\max \{g(\mathbf{h}, \omega, p), 0\}$ with a smooth function $g_{\epsilon}(\mathbf{h}, \omega, p)$ where

$$
g_{\epsilon}(\mathbf{h}, \omega, p)= \begin{cases}0 & \text { if } g(\mathbf{h}, \omega, p)<-\epsilon \\ \frac{(g(\mathbf{h}, \omega, p)+\epsilon)^{2}}{4 \epsilon} & \text { if }-\epsilon \leq g(\mathbf{h}, \omega, p) \leq \epsilon \\ g(\mathbf{h}, \omega, p) & \text { if } g(\mathbf{h}, \omega, p)>\epsilon\end{cases}
$$

and $\epsilon$ is a small positive number. The function in (9) is now approximated by the continuously differentiable functions $G_{\epsilon}(\mathbf{h})$ given by

$$
G_{\epsilon}(\mathbf{h})=\int_{\mathcal{P}} \int_{\Omega} g_{\epsilon}(\mathbf{h}, \omega, p) d \omega d p .
$$

The approach proposed in [10] will be used. More specifically, we use the concept of the penalty function to append the smoothen function (11) to the cost function in (6), forming a sequence of approximate optimization problems stated as follows:

$$
\min _{\mathbf{h} \in \mathcal{R}^{M N} \times 1} f_{\epsilon, \gamma}(\mathbf{h})
$$

where

$$
f_{\epsilon, \gamma}(\mathbf{h})=E(\mathbf{h})+\gamma G_{\epsilon}(\mathbf{h})
$$

and $\gamma$ is a penalty parameter. For each $\gamma$ and $\epsilon$, let the problem be referred to as Problem $\mathrm{P}_{\epsilon, \gamma}$.

Remark III.1: We have the following properties [13]: For every $\epsilon>$ 0 , there exists a $\gamma(\epsilon)>0$ such that for any $\gamma>\gamma(\epsilon)$, a solution of Problem $\mathrm{P}_{\epsilon, \gamma}$ will satisfy the continuous inequality constraints of Problem (6). In addition, suppose that $\mathbf{h}^{*}$ and $\mathbf{h}_{\epsilon, \gamma}^{*}$ are, respectively, the global optimal solutions of Problems (6) and Problem $\mathrm{P}_{\epsilon, \gamma}$ with $\gamma>\gamma(\epsilon)$. Then, the cost function $f_{\epsilon, \gamma}\left(\mathbf{h}_{\epsilon, \gamma}^{*}\right)$ approaches to $E\left(\mathbf{h}^{*}\right)$ as $\epsilon \rightarrow 0$.

In practice, we only consider a finite sequence of $\epsilon$. The solution to the optimization problem (6) is obtained by solving a sequence of unconstrained optimization problems $\mathrm{P}_{\epsilon, \gamma}$ with $\gamma$ chosen iteratively so that the continuous inequality constraint in (6) is satisfied. A procedure to search for a minimizer of the problem is presented as follows.

Procedure III.1: Check for a feasible solution to the problem (6).

- Solve the minmax optimization problem

$$
\begin{aligned}
& \min \epsilon \\
& \text { subject to } \\
& \frac{1}{e(\omega, p)}\left|H(\omega, p)-H_{d}(\omega, p)\right| \leq \epsilon, \forall \omega \in \Omega, p \in \mathcal{P}
\end{aligned}
$$

by using the semi-infinite linear programming approach or semidefinite programming (see, e.g., [14]). If the above problem ha a feasible solution with $\epsilon<1$, then this optimization problem has a feasible solution. This solution can be used as the initial solution to the Procedure III.1. Otherwise, the problem does not have a feasible solution and the problem needs to be reformulated.

Procedure III.2: Optimize Problem (6).

- Step 1: Initialize $\epsilon$ and $\gamma$.

- Step 2: Obtain the solution $\mathbf{h}_{\epsilon, \gamma}^{*}$ of Problem $\mathrm{P}_{\epsilon, \gamma}$.

- Step 3: If $\mathbf{h}_{\epsilon, \gamma}^{*}$ satisfies the continuous inequality constraints in (6), then go to Step 4. Otherwise, increase $\gamma$ and return to Step 2.

- Step 4: Decrease the value of $\epsilon$. If the difference in $E(\mathbf{h})$ between the two consecutive iterations is small then stop the procedure. Otherwise, the current optimal solution will be used as the initial to the next iteration and return to Step 2.

Remark III.2: The process of increasing $\gamma$ is finite by virtue of Remark III.1. 
As Problem $\mathrm{P}_{\epsilon, \gamma}$ is nonlinear and nonconvex, it may have many local minima. In the following section, a global optimization method incorporating a filled function is employed for solving Problem $\mathrm{P}_{\epsilon, \gamma}$ in Step 2 of Procedure III.2.

\section{OPTIMIZATION METHOD USING THE FILLED FunCTION}

As in [13], from a local minimizer $\mathbf{h}_{\epsilon, \gamma}^{*}$ of Problem $\mathrm{P}_{\epsilon, \gamma}$, a filled function is constructed so that when minimizing this filled function, a point with a lower cost value than $f_{\epsilon, \gamma}\left(\mathbf{h}_{\epsilon, \gamma}^{*}\right)$ is obtained. However, a more refined filled function will be used. More specifically, as the cost function $f_{\epsilon, \gamma}\left(\mathbf{h}_{\epsilon, \gamma}^{*}\right)$ is positive and relatively small for our filter design problem, we modify the quasi-filled function in [11] as follows:

$$
\begin{aligned}
F\left(\mathbf{h}, \mathbf{h}_{\epsilon, \gamma}^{*}, q, r, c\right) & \\
= & \exp \left(-\frac{\left\|\mathbf{h}-\mathbf{h}_{\epsilon, \gamma}^{*}\right\|^{2}}{q}\right) g_{r, c}\left(\frac{f_{\epsilon, \gamma}(\mathbf{h})-f_{\epsilon, \gamma}\left(\mathbf{h}_{\epsilon, \gamma}^{*}\right)}{f_{\epsilon, \gamma}\left(\mathbf{h}_{\epsilon, \gamma}^{*}\right)}\right) \\
& +h_{r, c}\left(\frac{f_{\epsilon, \gamma}(\mathbf{h})-f_{\epsilon, \gamma}\left(\mathbf{h}_{\epsilon, \gamma}^{*}\right)}{f_{\epsilon, \gamma}\left(\mathbf{h}_{\epsilon, \gamma}^{*}\right)}\right)
\end{aligned}
$$

where $q, r$ and $c$ are the filled function parameters

$$
g_{r, c}(t)= \begin{cases}c & t \geq 0 \\ -\frac{2 c}{r^{3}} t^{3}-\frac{3 c}{r^{2}} t^{2}+c & -r<t \leq 0 \\ 0 & \text { otherwise }\end{cases}
$$

and

$$
h_{r, c}(t)= \begin{cases}2 c & t \geq 1+r \\ 1-\frac{4 c-2}{r^{3}} t^{3}+\frac{(6 c-3)(r+2)}{r^{3}} t^{2}- & \\ \frac{(6 c-3)(2+2 r)}{r^{3}} t+\frac{4 c-2+(6 c-3) r}{r^{3}} & 1 \leq t \leq 1+r \\ 1 & 0<t<1 \\ \frac{r-2}{r^{3}} t^{3}+\frac{r-3}{r^{2}} t^{2}+1 & -r<t \leq 0 \\ t+r & t \leq-r .\end{cases}
$$

The proposed optimization method utilizes the following steps.

Procedure IV.1: Use for Step 2 of Procedure III.2. Search for the optimum $\mathbf{h}_{\epsilon, \gamma}^{*}$ of Problem $\mathrm{P}_{\epsilon, \gamma}$.

- Step 1: Initialize $\mathbf{h}^{0} \in \mathcal{R}^{M N \times 1} ; q, c$ and their upper limits; $r$ and its lower limit. Denote by $\mathcal{U}$ a set of unit directions, $\mathcal{U}=$ $\left\{\mathbf{u}_{1}, \cdots, \mathbf{u}_{K}\right\}$, where $K \geq M N$.

- Step 2: Obtain a local minimizer $\mathbf{h}_{\epsilon, \gamma}^{*}$ of Problem $\mathrm{P}_{\epsilon, \gamma}$ from the initial $\mathbf{h}^{0}$ by using a gradient based method.

- Step 3: Choose a unit direction in $\mathcal{U}$. From the point $\mathbf{h}_{\epsilon, \gamma}^{*}$, search along the unit direction for a point $\mathbf{h}_{\epsilon, \gamma}^{0}$ that satisfies

$$
\begin{aligned}
& f_{\epsilon, \gamma}\left(\mathbf{h}_{\epsilon, \gamma}^{0}\right) \leq f_{\epsilon, \gamma}\left(\mathbf{h}_{\epsilon, \gamma}^{*}\right) \\
\text { or } \quad & f_{\epsilon, \gamma}\left(\mathbf{h}_{\epsilon, \gamma}^{*}\right)<f_{\epsilon, \gamma}\left(\mathbf{h}_{\epsilon, \gamma}^{0}\right)<f_{\epsilon, \gamma}\left(\mathbf{h}_{\epsilon, \gamma}^{*}\right)+1 .
\end{aligned}
$$

Construct the filled function $F\left(\mathbf{h}, \mathbf{h}_{\epsilon, \gamma}^{*}, q, r, c\right)$ and minimize this function using a local search method starting from the initial point $\mathbf{h}_{\epsilon, \gamma}^{0}$. If a point with a lower minimum is obtained then set $\mathbf{h}^{0}$ as this point and return to Step 2. Otherwise, increase either $q, c$ or reduces $r$ and re-optimize the filled function. Stop the procedure when the values of $q, r$ and $c$ reach their allowable limits and all the unit directions have been chosen. Set $\mathbf{h}_{\epsilon, \gamma}^{*}$ as the global minimizer of the optimization problem.

Note that if all the unit directions in $\mathcal{U}$ have been used and no better point is found, we may increase the number of search directions and continue with the process using the new directions. We will regard the solution obtained as a global optimal solution if the process does not give rise to a point in a lower basin after all the directions have been utilized. Clearly, we may miss finding the global optimizer unless the number of searched directions is very large, but this becomes

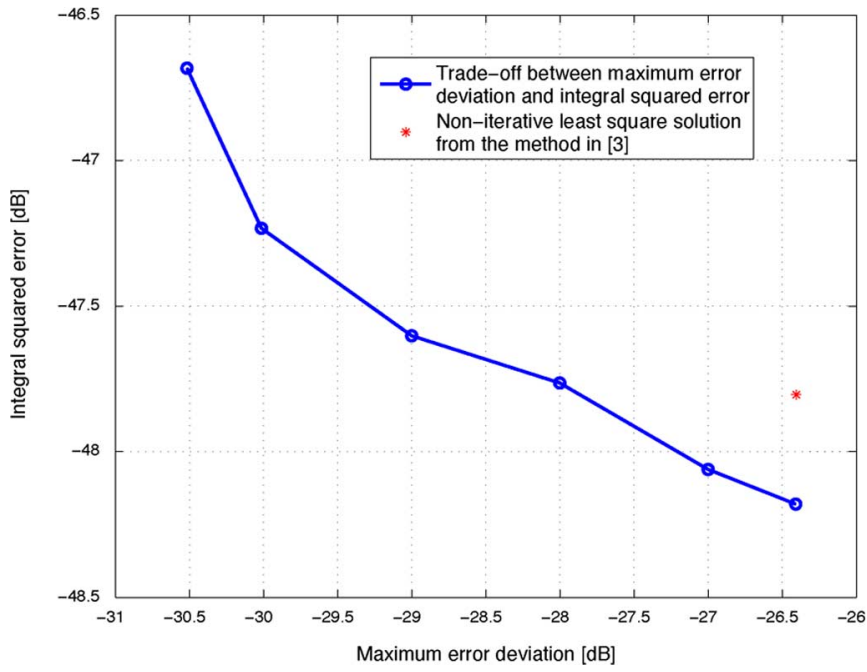

Fig. 1. Tradeoff plot between maximum error deviation and integral squared error for VFD allpass filter.

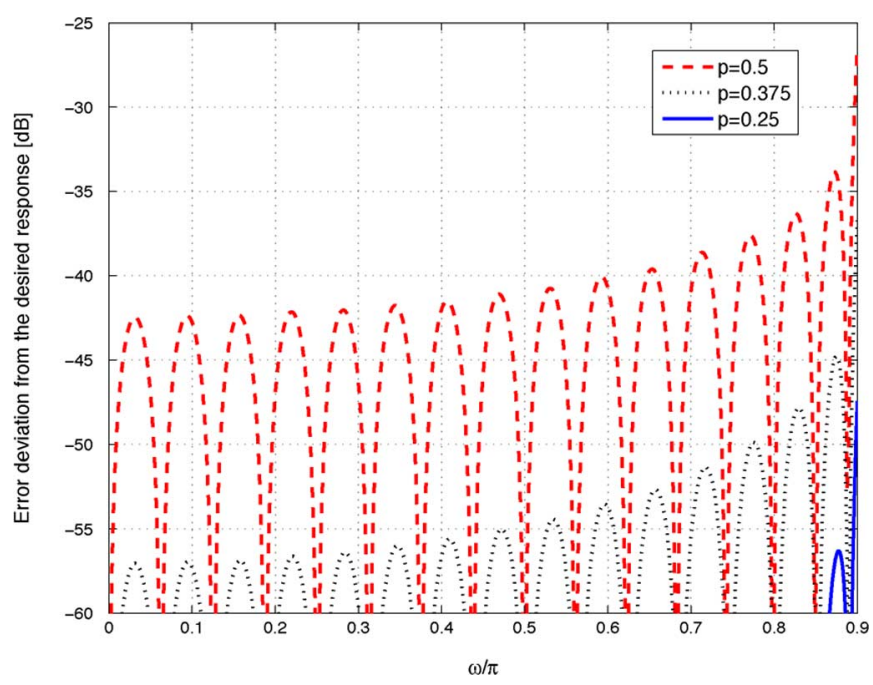

Fig. 2. Noniterative LS design solution for the VFD allpass filter with $p=$ $\{0.5,0.375,0.25\}$. The VFD allpass filter has maximum error deviation of $-26.4050 \mathrm{~dB}$ and integral squared error of $-47.8047 \mathrm{~dB}$.

impractical even for problems of moderate size. Our extensive simulation studies suggest that good quality optimal solutions can often be obtained without having to take the number of directions to be excessively large.

\section{DESIGN EXAMPLE}

Consider the design of an allpass VFD filter with $N=15, M=4$ and $\alpha=0.9 \mathrm{~dB}$. The weighting function $W(\omega, p)$ is one for all $\omega$ and $p$.

For Procedure III.2, the initial values for $\epsilon, \gamma$ are chosen, respectively, as 0.01 and 100. Also, for Procedure IV.1, the upper limit for $q$ and $c$ is $10^{8}$ while the lower limit for $r$ is $10^{-8}$. The initial values of $q, r$ and $c$ are chosen as $q=r=10^{2}$ and $c=10^{-2}$. The number of discretized points for $\omega$ is 256 while the number of discretized points for $\delta$ is 128 .

Fig. 1 plots the integral square error $20 \log _{10} E(\mathbf{h})$ [dB] for different maximum error deviation from the desired response. The point "*" in the plot shows the least square (LS) solution; see, e.g., [3]. Denote by $e_{\mathrm{LS}}$ the maximum error deviation for this solution. The plot shows the 


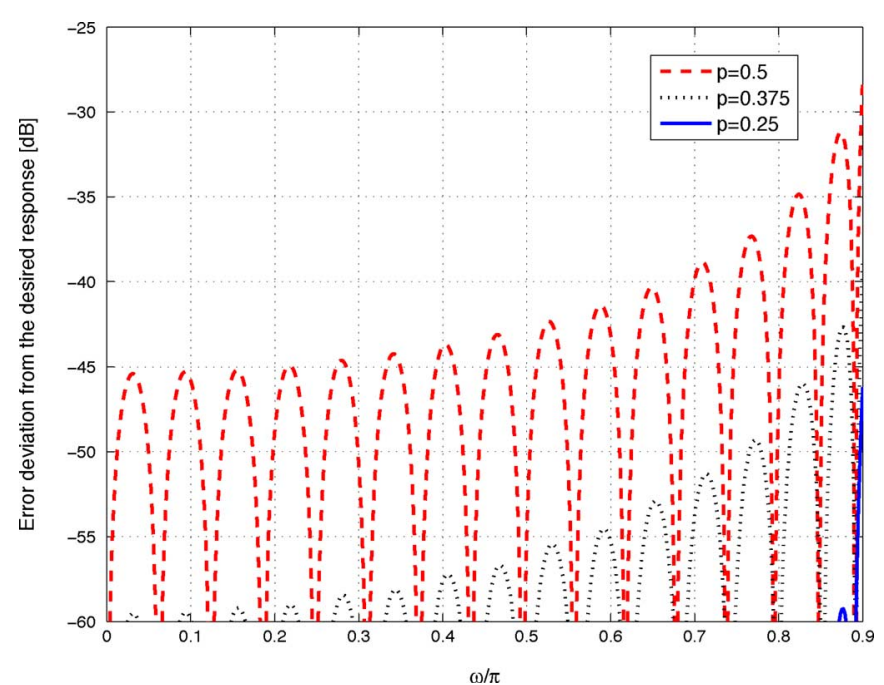

Fig. 3. VFD allpass filter with $p=\{0.5,0.375,0.25\}$. The VFD allpass filter has maximum error deviation of $-28.0013 \mathrm{~dB}$ and integral squared error of $-47.7643 \mathrm{~dB}$.

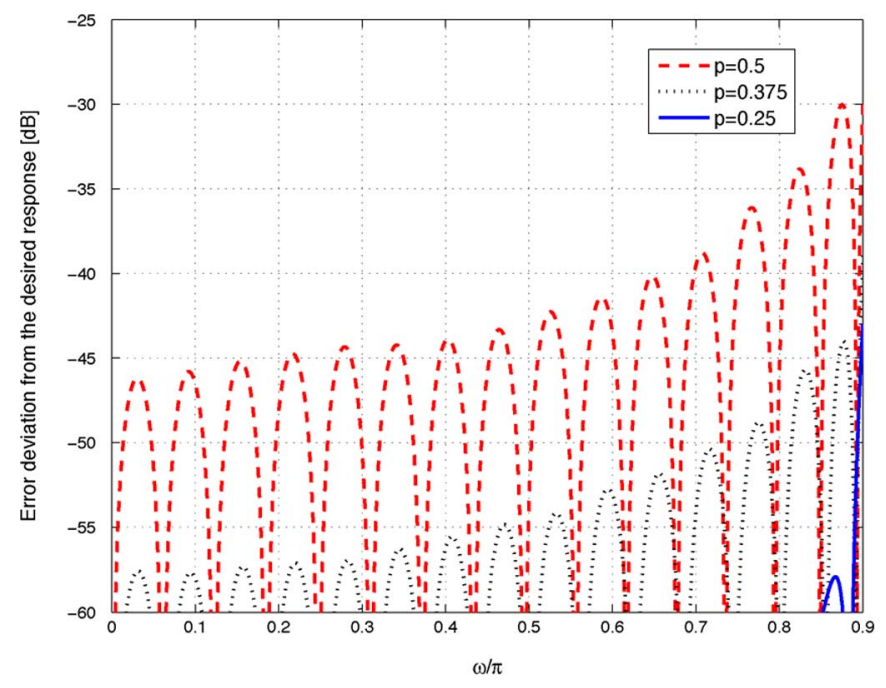

Fig. 4. VFD allpass filter with low maximum error deviation and $p=\{0.5,0.375,0.25\}$. The VFD allpass filter has maximum error deviation of $-30.0145 \mathrm{~dB}$ and integral squared error of $-47.2325 \mathrm{~dB}$.

trade-off between the integral squared error and the maximum error deviation. In addition, from the LS solution, the maximum error deviation can be reduced while maintaining approximately the same integral squared error. Thus, this formulation allows a flexible design to control the maximum error deviation for the allpass VFD filter.

Fig. 2 plots the frequency response error deviation from the desired response, expressed as

$$
20 \log _{10}\left|H(\mathbf{h}, \omega, p)-H_{d}(\omega, p)\right|
$$

for the noniterative LS solution (for example, the one obtained in [3]) for a few values of the tuning parameter $p$, including the extreme value 0.5 . The VFD allpass filter has a maximum error deviation of -26.40 $\mathrm{dB}$ and an integral squared error of $-47.80 \mathrm{~dB}$.

Fig. 3 plots the frequency response error deviation from the desired responses of an allpass VFD filter. The maximum error deviation of the filter is approximately $1.6 \mathrm{~dB}$ lower than that of the LS filter, while

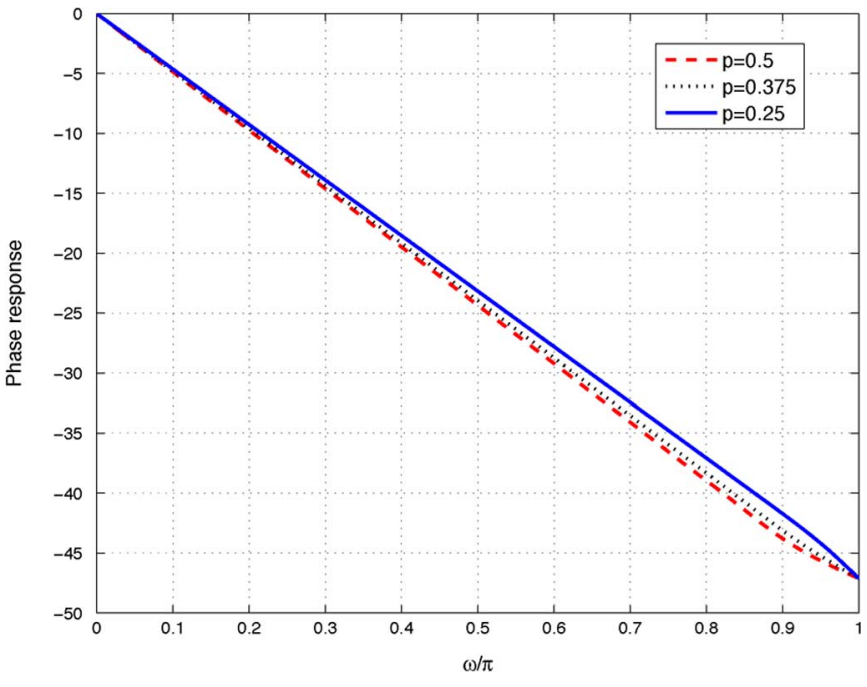

Fig. 5. Phase response for different VDF allpass filters. The VFD filter has maximum error deviation of maximum error deviation of $-28.0013 \mathrm{~dB}$ and integral squared error of $-47.7643 \mathrm{~dB}$.

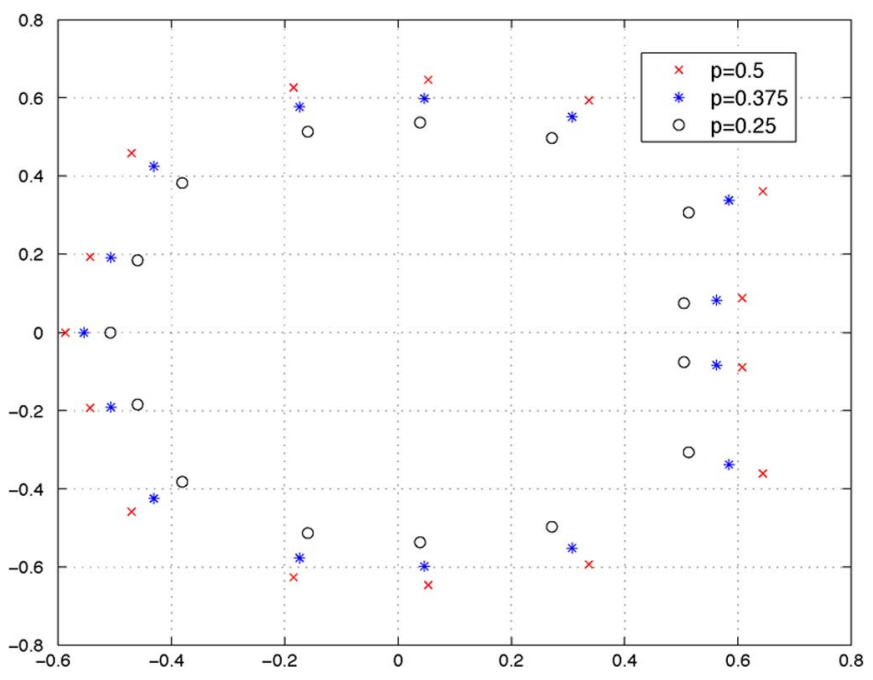

Fig. 6. Poles for different VDF allpass filters. The VFD filter has maximum error deviation of maximum error deviation of $-28.0013 \mathrm{~dB}$ and integral squared error of $-47.7643 \mathrm{~dB}$.

maintaining approximately the same integral squared error. More specifically, the VFD allpass filter has maximum error deviation of $-28.0013 \mathrm{~dB}$ and integral squared error of $-47.7643 \mathrm{~dB}$. The plot is shown for $p=\{0.5,0.375,0.25\}$.

Fig. 4 plots the frequency response error deviation from the desired response for the allpass filter with a low maximum error deviation. The VFD allpass filter has a maximum error deviation of $-30.0145 \mathrm{~dB}$ and an integral squared error of $-47.2325 \mathrm{~dB}$. The maximum error deviation is lower than those reported in the previous two cases, depicted in Figs. 2-3. There is only a small increased in the integral squared error.

For completeness, Figs. 5 and 6 show, respectively, an example of the phase response and the pole positions of the allpass VFD filters with maximum error deviation of $-28.0013 \mathrm{~dB}$ and integral squared error of $-47.7643 \mathrm{~dB}$. As noted earlier, the phase responses of all the allpass filters at $\pi$ are $\theta(\pi, p)=-15 \pi$ for all $p \in[-0.5,0.5]$. Thus, the allpass filters are stable which can also be seen in Fig. 6 with all the poles well inside the unit circle. 


\section{CONCLUSION}

In this correspondence, a general design for allpass variable fractional delay (VFD) digital filters with minimum weighted integral squared error subject to a constraint on maximum error deviation from the desired response was formulated. Design examples show that a trade-off can be achieved between the integral squared error and the maximum error deviation for the allpass VFD filters. From the WLS solution, the maximum error deviation can be reduced while maintaining approximately the same integral squared error.

\section{ACKNOWLEDGMENT}

The authors would like to thank Prof. A. Cantoni for useful discussions about allpass VFD filter structure.

\section{REFERENCES}

[1] C. W. Farrow, "A continuously variable digital delay element," in Proc. IEEE Int. Symp. Circuits Syst., Jun. 1988, vol. 3, pp. 2641-2645.

[2] S. C. Pei and C. C. Tseng, "A comb filter design using fractionalsample delay," IEEE Trans. Circuits Syst. II, vol. 45, no. 6, pp. 649-653, Jun. 1998.

[3] T. B. Deng, "Noniterative WLS design of allpass variable fractionaldelay digital filters," IEEE Trans. Circuits Syst., vol. 53, no. 2, pp. 358-371, Feb. 2006.

[4] H. H. Dam, A. Cantoni, K. L. Teo, and S. Nordholm, "FIR variable digital filter with signed power-of-two coefficients," IEEE Trans. Circuits Syst. I, vol. 54, no. 6, pp. 1348-1357, Jun. 2007.

[5] H. H. Dam, A. Cantoni, K. L. Teo, and S. Nordholm, "Vatiable digital filter with least square criterion and peak gain constraints," IEEE Trans. Circuits Syst. II, vol. 54, no. 1, pp. 24-28, Jan. 2007.

[6] M. Makundi, T. I. Laakso, and V. Valimaki, "Efficient tunable IIR and allpass structures," Electron. Lett., vol. 37, pp. 344-345, Mar. 2001.

[7] C. C. Tseng, "Design of 1-D and 2-D variable fractional delay allpass filters using weighted least square methods," IEEE Trans. Circuits Syst. I, vol. 49, no. 10, pp. 1413-1422, Oct. 2002.

[8] Z. Jing, "A new method for digital all-pass filter design," IEEE Trans. Acoust., Speech, Signal Process., vol. 35, pp. 1557-1564, Nov. 1987.

[9] J. Y. Kaakinen and T. Saramaki, "An algorithm for the optimization of adjustable fractional delay all-pass filters," in Proc. IEEE ISCAS, Vancouver, QC, Canada, May 23-26, 2006, vol. III, pp. 153-156.

[10] K. L. Teo, V. Rehbock, and L. S. Jennings, "A new computational algorithm for functional inequality constrained optimization problems," Automatica, vol. 29, no. 3, pp. 780-792, 1993.

[11] Z. Y. Wu, H. W. J. Lee, L. S. Zhang, and X. M. Yang, "A novel filled function method and quasi-filled function method for global optimization," Comput. Optim. Appl., vol. 34, pp. 249-272, 2005.

[12] A. T. Chotterra and G. A. Jullien, "A linear programming approach to recursive digital filter design with linear phase," IEEE Trans. Circuits Syst., vol. CAS-29, no. 3, pp. 139-149, Mar. 1982.

[13] C. Z. Wu, K. L. Teo, V. Rehbock, and H. H. Dam, "Global optimum design of uniform FIR filter bank with magnitude constraints," IEEE Trans. Signal Process., vol. 56, no. 11, pp. 5478-5486, Nov. 2008.

[14] C. K. S. Pun and S. C. Chan, "Minmax design of digital all-pass filters with prescribed pole radius constraint using semidenite programming," in Proc. Int. Conf. Acoustics, Speech, Signal Processing (ICASSP), 2003, pp. 413-416.

\section{Two-Channel Linear Phase FIR QMF Bank Minimax Design via Global Nonconvex Optimization Programming}

Charlotte Yuk-Fan Ho, Bingo Wing-Kuen Ling, Lamia Benmesbah, Ted Chi-Wah Kok, Wan-Chi Siu, and Kok-Lay Teo

\begin{abstract}
In this correspondence, a two-channel linear phase finite-impulse-response (FIR) quadrature mirror filter (QMF) bank minimax design problem is formulated as a nonconvex optimization problem so that a weighted sum of the maximum amplitude distortion of the filter bank, the maximum passband ripple magnitude and the maximum stopband ripple magnitude of the prototype filter is minimized subject to specifications on these performances. A modified filled function method is proposed for finding the global minimum of the nonconvex optimization problem. Computer numerical simulations show that our proposed design method is efficient and effective.
\end{abstract}

Index Terms-Filled function, global optimization, nonconvex optimization problem, two-channel linear phase FIR QMF bank minimax design.

\section{INTRODUCTION}

Since transition bandwidths of the filters in two-channel filter banks are usually larger than those in multichannel filter banks, lengths of the filters in two-channel filter banks are usually shorter than those in multichannel filter banks. Moreover, as only a single prototype filter is required for the design of a quadrature mirror filter (QMF) bank and all other filters are derived from the prototype filter, the total number of filter coefficients required for the design of a QMF bank is usually smaller than those in general filter banks. Furthermore, as the linear phase property of the filters guarantees no phase distortion of the filter bank and the FIR property of the filters guarantees the bounded input bounded output stability of the filter bank, two-channel linear phase FIR QMF banks find many applications in image and video signal processing [1].

Unlike a multichannel QMF bank [2], [3], a two-channel QMF bank could not achieve the exact perfect reconstruction with the prototype filter having very good frequency selectivity [4]. Hence, it is useful to

Manuscript received September 28, 2009; accepted April 18, 2010. Date of publication April 26, 2010; date of current version July 14, 2010. The associate editor coordinating the review of this manuscript and approving it for publication was Prof. Jean-Christophe Pesquet. The work obtained in this correspondence was supported by a research grant (project G-YD26) from The Hong Kong Polytechnic University, the Centre for Multimedia Signal Processing, The Hong Kong Polytechnic University, the CRGC grant (project PolyU 5105\01E) from the Research Grants Council of Hong Kong, as well as a research grant from the Australian Research Council.

C. Y.-F. Ho is with the School of Mathematical Sciences, Queen Mary, University of London, London, E1 4NS, U.K. (e-mail: c.ho@qmul.ac.uk).

B. W.-K. Ling is with the School of Engineering, University of Lincoln, Brayford Pool, Lincoln, Lincolnshire, LN6 7TS, U.K. (e-mail: wling@lincoln.ac. uk).

L. Benmesbah is with the Department of Electronic Engineering, Division of Engineering, King's College London, Strand, London, WC2R 2LS, U.K. (e-mail: lamia.benmesbah@kcl.ac.uk).

T. C.-W. Kok is with Canaan Microelectronics, Kowloon, Hong Kong, China (e-mail: eekok@ieee.org).

W.-C. Siu is with the Department of Electronic and Information Engineering, Hong Kong Polytechnic University, Kowloon, Hong Kong, China (e-mail: enwcsiu@polyu.edu.hk).

K.-L. Teo is with the Department of Mathematics and Statistics, Curtin University of Technology, Perth, CRICOS Provider Code 00301J, Australia (e-mail: K.L.Teo@curtin.edu.au).

Color versions of one or more of the figures in this correspondence are available online at http://ieeexplore.ieee.org.

Digital Object Identifier 10.1109/TSP.2010.2049107 